\title{
In silico-mediated virtual screening and molecular docking platforms for discovery of non $\beta$-lactam inhibitors of y-49 $\beta$-lactamase from Mycobacterium tuberculosis
}

\begin{abstract}
Tuberculosis (TB) is caused by Mycobacterium tuberculosis, and remains a major, worldwide health concern. The emergence of $M$. tuberculosis strains that are resistant to front line antimicrobacterial drugs such as isoniazid and rifampicin have greatly complicated efforts to control the spread of TB. Of the various resistance mechanisms, the most effective involves the production of $\beta$-lactamases that hydrolyze $\beta$-lactam antibiotics. One approach to overcoming resistance to $\beta$-lactam antibiotics is to find new drugs or additives that can prevent the $\beta$-lactam antibiotic from being hydrolyzed by $\beta$-lactamases. The spectrum of anti-TB drugs consisting of non $\beta$-lactam scaffolds has been expanded by the development of new boronic acid derivatives and cationic peptidic inhibitors that proved effective in neutralizing bacterial resistance, especially when administered in combination with antibiotics. The intrinsic resistance to $\beta$-lactam antibiotics was due mainly to the presence of a chromosomally-encoded gene (blaC) in M. tuberculosis for a Class A, Ambler $\beta$-lactamase $(\mathrm{BlaC})$. The $\mathrm{BlaC}$ enzyme has already been validated as one of the leading targets of tuberculosis therapy. In the search for new $\beta$-lactamase inhibitors we employed the on-line server Pharmit (pharmit.csb.pitt.edu) to discover new 3-D pharmacophores and search different databases of chemical libraries, and commercial compounds. Autodock Vina was employed to perform molecular docking of selected compounds from ChEMBL/ MolPort/ZINC databasesusingthe M. tuberculosis $\beta$-lactamase 3M6B.pdb as the target protein. This combination of 3-D pharmacophore screening and molecular docking lead to the discovery of natural products, and novel, small molecules inhibitors of Y-49ß-lactamase from Mycobacterium tuberculosis. Notably new 3D-pharmacophores derived from hydroxyphenylglycine and fragments from the family of biphenyls, aromatic ketones, and stilbenes emerged as potential inhibitors of the recombinant Y-49 $\beta$-lactamase exhibiting in vitro inhibitory constants (Ki), in the low $5 \mathrm{uM}-200 \mathrm{uM}$ range. These newly discovered pharmacophore features enabled by in silico screening are extending the chemical space for discovery of novel and potent inhibitors of $\beta$-lactamases.
\end{abstract}

Keywords: non $\beta$-lactam scaffolds inhibitors, y-49 $\beta$-lactamase, 3-D pharmacophores Pharmit, molecular docking, autodock vina
Volume 7 Issue I - 2018

\author{
Janet Gonzalez,' Elainie Lendebol,' \\ Angela Shen,' Manfred Philipp, ${ }^{2}$ Cristina C \\ Clement ${ }^{2,3}$ \\ 'Department of Natural Sciences, LaGuardia Community \\ College, USA \\ ${ }^{2}$ Department of Chemistry, Lehman College of the City \\ University of New York, USA \\ ${ }^{3}$ Pathology Department, Albert Einstein College of Medicine, \\ USA
}

\begin{abstract}
Correspondence: Janet Gonzalez, Natural Sciences, LaGuardia Community College, Long Island City, New York, United States,
\end{abstract} USA,Email jagonzalez@lagonzalez@lagcc.cuny.edu

Cristina C. Clement, Pathology Department, Albert Einstein College of Medicine, Bronx, NY, I046I, USA; and Department of Chemistry, Lehman College of the City University of New York, Bronx, New York, 10468, USA, Tel 7184303468,

Email cristina.clement@einstein.yu.edu

Received: May 05, 2017 | Published: August II, 2017
Abbreviations: TB, tuberculosis; Mtb, mycobacterium tuberculosi; SBDD, structure-based drug discovery

\section{Introduction}

Tuberculosis (TB), caused by Mycobacterium tuberculosis (Mtb), is a worldwide health concern. The world health organization in its 2017 tuberculosis report states, "TB is the ninth leading cause of death worldwide and the leading cause from a single infectious agent, ranking above HIV/AIDS". ${ }^{1-3}$ The failure to control TB is due to the emergence of Mtbstrains that are resistant to first line beta lactam antibiotics, because of overuse. One of the most effective resistance mechanisms to $\beta$-lactam antibiotics involves the production of $\beta$-lactamases which cleave the amide bond in the target $\beta$-lactam ring hydrolyzing the drug before it reaches its target. The beta-lactamases are classified into four classes: A, B, C and D. These classes are based on conserved and distinguishing amino acid motifs. ${ }^{1-3}$ Classes A, C, and $\mathrm{D}$ include enzymes that hydrolyze their substrates by forming an acyl enzyme through an active site serine. Class B $\beta$-lactamases are metalloenzymes that utilize at least one active-site zinc ion to facilitate $\beta$-lactam hydrolysis. One of the most efficient and prevalent mechanisms of resistance to $\beta$-lactam antibiotics is the production of $\beta$-lactamases in both Gram-negative and Gram-positive bacteria that hydrolyze the drugs before they can reach their target and exert the desired effect. These resistance mechanisms are important, and each bacterium can create a combination of defenses depending on the selective pressures placed on it. ${ }^{1-4}$ The intrinsic resistance to $\beta$-lactam antibiotics was demonstrated to be mainly due to the presence of a chromosomally-encoded gene (blaC) in M. tuberculosis for a Class $\mathrm{A}$, Ambler $\beta$-lactamase $(\mathrm{BlaC})$. The $\mathrm{BlaC}$ enzyme has already been validated as one of the leading targets of tuberculosis therapy. This enzyme is extremely active against penicillins and cephalosporins, but weaker against carbapenems. ${ }^{5-7}$ The $\mathrm{BlaC}$ enzyme can be inhibited by clavulanate, avibactam, and boronic acids..$^{5-10}$ The first $\beta$-lactamase inhibitor to be used clinically was clavulanic acid, from Streptomyces clavuligerus. When combined with traditional $\beta$-lactams such as amoxicillin and ticarcillin, clavulanic acid produces a broad-spectrum of antibiotic combinations effective against many $\beta$-lactamaseproducing bacteria. ${ }^{5-7}$ 
The success of clavulanic acid established $\beta$-lactamase inhibitors as one solution to the problem of $\beta$-lactam antibiotic-resistant bacteria. To date only four $\beta$-lactamase inhibitors have been approved by the Food and Drug Administration and are being clinically used: clavulanic acid; the two penicillanic acid sulfones (tazobactam and sulbactam); and most recently, avibactam. However, resistance has emerged even to these potent antibiotic combinations among formerly susceptible bacteria. ${ }^{6-10}$ Research studies in the last decade have shown that the most promising approach to overcoming resistance to $\beta$-lactam antibiotics requires high throughput screening of new non $\beta$-lactam scaffolds, those demonstrating inhibitory activity against a large family of $\beta$-lactamases, in addition to the improvement of established antibiotics. ${ }^{11-15}$

This research aimed to discover new $\beta$-lactamase inhibitors by employing a combination of molecular docking and biochemical kinetics assays. We first focused on discovery of novel 3D-pharmacophores and fragments from the family of diphenyl, aromatic ketone, hydroxy-phenyl derivatives and stilbene, all previously described by others to exhibit antibacterial activity. ${ }^{16-19}$ The in vitro screening platform, using kinetics of $\beta$-lactamase inhibition with nitrocefin substrate, enabled the discovery of potential inhibitors of Y-49 $\beta$-lactamase exhibiting inhibitory constants, Ki, in the lowmM range. The finding of these novel pharmacophore features exhibiting anti- $\beta$-lactamase inhibition offered additional strength to the structure-based drug design (SBDD) approach by selectively defining the chemical space of the ligand libraries during in silico screening using the on-line server Pharmit (pharmit.csb.pitt.edu) ${ }^{20}$ Specifically, we searched different databases of chemical librariesand commercial compounds such as ChEMBL/MolPort/ZINC ${ }^{21}$ where the pharmacophore features of the ligands could be chosen to resemble the chemical space of selected stilbenes and other small organic compounds discovered to inhibit the Y-49 $\beta$-lactamase using the in vitro, enzymatic screening assay. This strategy defined the in silico targeted screening approach for ligand profiling, and the discovery of Y-49 $\beta$-lactamase active site binders. An independent in silico screening approach used a wider chemical space for ligand profiling, albeit constrained by their physical chemical properties as defined by the Lipinski rule. ${ }^{22}$ Autodock Vina ${ }^{23}$ was employed to perform molecular docking of selected compounds from databases using the M. tuberculosis $\beta$-lactamase 3M6B.pdb as the target protein.

This research highlights the importance of coupling the validation assays for the enzyme inhibition or ligand binding to the target protein with the optimized platform for in silico screening of 3-D pharmacophores and molecular docking, specifically tailored for each molecular target of interest. The data presented herein support the need for multiple rounds of docking and minimization of the type allowed in Pharmit. This approach improved the discovery of novel 3D-pharmacophore features, and in turn enabled drug discoveries using the SBDD method. Additionally, the new docking platforms described here may provide new avenues for overcoming $\beta$-lactamasebased resistance that remains a key clinical challenge.

\section{Materials and methods}

Nitrocefin substrate for $\beta$-lactamase was purchased from Calbiochem, CA. Unless otherwise noted, all other chemicals, including the compounds used in kinetics for $\beta$-lactamase inhibition, were obtained from Sigma-Aldrich (St. Louis, MO).

\section{Methods}

\section{Bioinformatics analysis of $Y-49 \beta$-lactamase}

The FASTA sequence of Y-49 $\beta$-lactamase was subjected to sequence similarity search using BLASTP algorithm ${ }^{24}$ and the protein hits with more than $99 \%$ sequence identity were further searched against the Brookhaven RCSB PDB database for finding of additional proteins with sequence and structural identity using the VAST $(+)$ algorithm andmMDB database. ${ }^{25}$ The top ten hits displaying more than $99 \%$ sequence identity and less than $0.25 \AA$ RMSD for their structural similarity were identified and further analyzed for the resolution and accuracy of their X-Ray structure.

\section{Preparation of target 3M6B protein structure for analysis of protein-ligand interactions using the Molecular Operating Environment (MOE)}

Molecular modeling was initially performed with MOE (from CCG, Montreal, Canada) to extract the main pharmacophore features that define the main interactions between the ligand ertapenem (RG1) and $\beta$-lactamase protein in the 3M6B.pdb structure, reported by John Blanchard group at Albert Einstein College Inc. ${ }^{26}$ The MOE automatically corrected the crystallographic data, including missing loops, empty residues, chain termini or breaks, etc. using the "Structure Preparation" application. The hydrogen bonding network wand the individual atomic protonation states were optimized using the "Protonate3D" module. ${ }^{27-29}$ The built-in 2D-diagram option was used to visualize the amino acid residues in close contact with the RG1 (ertapenem) ligand, and to identify the main hydrogen bonds, salt bridges, hydrophobic interactions, cation- $\pi$, and the ligand solvent exposure. The original pose of RG1 was minimized in the active site of $\beta$-lactamase using the Amber12: EHT force field provided by MOE that employs Amber12 parameters for macromolecules, and Extended Hückel Theory (EHT) parameterization for small molecules that accounts for electronic effects. ${ }^{29}$ The contribution of each protein residue-ligand atom interaction to the final reported free energy describing 3M6B/RG1 complex was extracted using the built-in MOE algorithm after minimization, and generation of a 2D-diagram for protein-ligand interaction (the RG1 is covalently adducted to the Ser 84 in the 3M6B structure).

Virtual screening of large compound databases and SBDD using online Pharmit server (http://pharmit.csb.pitt.edu)

To assist in the discovery of active site directed $\beta$-lactamase inhibitors the online comprehensive platform Pharmit was used for analyzing the best combination of pharmacophore features: hydrogen bond acceptor; hydrogen bond donor; hydrophobic; aromatics; and electrostatics. Accordingly, a predefined pharmacophore query was generated by Pharmit based on the analysis of the complex interaction between ertapenem (RG1) and $\beta$-lactamase, provided by the structure 3M6B.pdb. ${ }^{26}$ For each defined combination of pharmacophore features query, we selected and searched the pre-built compound libraries in Pharmit for matching compounds, including MolPort, CHEMBL21, ChemDiv, NCI Open Chemical Repository, PubMed and ZINC. A variety of filtering and ranking criteria were applied, including those combining drug-like properties, observing Lipinski's "rule of five". ${ }^{22}$ We chose any two combinations of the following: molecular mass (MW) less than $500 \mathrm{Da}$; high lipophilicity (expressed as $\log$ P less than 5); less than 5 hydrogen bond donors; less than 10 hydrogen bond acceptors and a topological surface area (TPSA) less than 200 (a measure of drug ability to permeate the cell membranes). ${ }^{22}$ The crystallographic water found in the active site of 3M6B structure was allowed by Pharmit to be part of the receptor active site. The hits were further refined and ranked using the energy minimization option provided in Pharmit that employs the AutoDock Vina ${ }^{23}$ scoring function $(\mathrm{kcal} / \mathrm{mol})$ and smina, a fork of AutoDock Vina with enhanced minimization functionality. ${ }^{20}$ The minimization procedure enabled the optimization of both the pose and conformation of the identified hits 
and improved the speed of structure-based drug discovery of novel, non-beta lactam scaffolds and compounds that are potential inhibitors of Y-49 $\beta$-lactamase.

The final hits were sorted according to predicted binding affinity inkcal $/ \mathrm{mol}$, eliminating poses with unfavorable binding energies (e.g. $>-8.0 \mathrm{kcal} / \mathrm{mol}$ (more negative values are more favorable). We also included an additional scoring function, namely the minimized RMSD (mRMSD) that is the RMSD between the query-aligned pose and the minimized pose. This score provides an indication of how far the compound has deviated from the original query. ${ }^{20}$ Minimized results were also thus sorted to exclude poses with significant deviations from the original query (e.g. $2 \AA$ ). We performed an independent analysis of the pharmacophore features to assess the contribution to the relative free energy of interaction between the ligand hits and 3M6B protein by employing Molegro Molecular Viewer (MMV), and Molegro Virtual Docker (MVD) software. In addition, the Molegro Dock software generated EPair energy terms, expressed as MolDock scoring functions. The MolDock scoring function consisted of mainly the pairwise (PLP) steric and hydrogen bonding energy of interaction between each lead compound and the amino acid residues from the active site of target $\beta$-lactamase protein (Depicted as chain A in Table 2).

\section{Bioinformatics of Y-49 $\beta$-lactamase primary sequence}

CLC bio Sequence Viewer (version 6.5, from QIAGEN) was used to create and edit the primary sequence alignments for selected $\beta$-lactamase proteins-class A-that are important pharmacological targets, such as those from the Mycobacterium, Streptomyces, and Bacillus species using the built-in progressive alignment algorithm tools. ${ }^{30}$ We selected eighteen Class A $\beta$-lactamase proteins that were known or potential pharmacological targets of interest for combating a wide range of bacterial infections, including bacteria with high resistance to antibiotics. ${ }^{12,15}$ The sequence logos for the identified conserved motifs in beta-lactamase proteins were displayed using the built-in algorithm provided by CLC bio Sequence Viewer. The overall height of each stack indicates the sequence conservation at that specific amino acid position; the height of symbols within each stack reflects the relative frequency of the corresponding amino acid. The three-dimensional visualization of amino acids identified in the four conserved motifs was performed by molecular graphics analysis provided by UCSF Chimera package from the Resource for Biocomputing, Visualization, and Informatics at the University of California, San Francisco (https://www.cgl.ucsf.edu/chimera/).

\section{Kinetics of Y-49 $\beta$-lactamase inhibition by different pharmacophore fragments and compounds}

We measured the ability of different pharmacophore fragments and compounds to inhibit the recombinant Y-49 $\beta$-lactamase. The cloning, expression, and purification of Y-49 $\beta$-lactamase was performed using a modified workflow described elsewhere, ${ }^{31}$ and is the subject of another upcoming manuscript. The host for the recombinant protein expression was E. Coli Top10 (Invitrogen), containing the pTrcHis B plasmid (Invitrogen), expressing Y-49 from Mycobacterium tuberculosis. We are indebted to Douglas S. Kernodle, M. D., Vanderbilt University School of Medicine, Division of Infectious Diseases, Nashville, TN for this gift. After determining the kinetic parameters for the recombinant Y-49 $\beta$-lactamase and nitrocefin substrate (i.e., $\mathrm{km}=95 \pm 10 \mu \mathrm{M}$ and $\mathrm{kcat}=15.34 \pm 0.5 \mathrm{sec}^{-1}$ ), we performed the inhibition assays using $24 \mu \mathrm{M}$ chromogenic nitrocefin substrate $\left(\xi_{486}=20500 \mathrm{M}^{-1} \mathrm{~cm}^{-1}\right)$ and $0.05-5 \mathrm{nM}$ enzyme concentration in a $200-250 \mu \mathrm{L}$ final volume of $25 \mathrm{mM}$ HEPES buffer, $\mathrm{pH}=7.4$. All kinetics assays were performed in 96 microplates, and different compounds were tested at different concentrations, ranging 0.5 $300 \mu \mathrm{M}$. A BIOTEK Synergy 4 multi detection microplate reader was used to record the data by monitoring the absorption at $486 \mathrm{nM}$. When present, the DMSO was kept below $5 \%$ in the final reaction mix. The reaction was initiated with the addition of the enzyme to the reaction mix containing the constant concentration of nitrocefin substrate, and different concentrations of the tested compound. The initial velocities (in $\mu \mathrm{M} / \mathrm{min}$ ) were calculated for the reaction run both in the absence (control $\mathrm{V}_{0}$ ) and the presence of different inhibitor concentrations (V). The graph plotting the initial velocity $(\mu \mathrm{M} / \mathrm{min})$ vs the concentration of inhibitor (in $\mu \mathrm{M}$ ) was used to determine the apparent inhibitory constant $\left(\mathrm{Ki}^{\text {(app) }}\right)$ by fitting the data to the Morrison equation provided by the GraphPad Prism 7.0 (GraphPad Software, La Jolla, California, USA). The following parameters were constrained during the fit with Morrison equation: $\mathrm{Km}=95 \mu \mathrm{M}$; $\mathrm{Et}=0.00005-0.005 \mu \mathrm{M}$ and $[\mathrm{S}]=24 \mu \mathrm{M}$. An independent assessment of $\mathrm{Ki}^{(\mathrm{app})}$ was performed by fitting the relative velocity (i.e. $\mathrm{Vr}=\mathrm{V} / \mathrm{V}_{0}$ ) vs the concentration of inhibitor (in $\mu \mathrm{M}$ ) and fitting the data to the equation $\mathrm{Ki}=[[\mathrm{I}]-[\mathrm{E}](\mathrm{Vr}-$ $1)] /(1 / \mathrm{Vr}-1)$ as described originally elsewhere. ${ }^{32}$ The experimental free energy of interaction (inkcal $/ \mathrm{mol}$ ) was calculated using the formula: $\mathrm{DG}=-\mathrm{RT} \operatorname{lnKeq}\left(\right.$ where $\mathrm{R}=-0.001987 \mathrm{kcal} . \mathrm{K}^{-1} \cdot \mathrm{mol}^{-1} ; \mathrm{T}=310 \mathrm{~K}\left(37^{\circ} \mathrm{C}\right)$; and $\mathrm{Keq}=1 / \mathrm{Ki})$.

\section{Statistics}

Statistical analysis was performed using Windows GraphPad Prism 7.0 (GraphPad Software, La Jolla, California, USA). Numerical results are reported as mean +/- SE or +/-SDV when appropriate. Unless stated otherwise, the data are derived from a minimum of three independent experiments.

\section{Results and discussion}

\section{Development of a platform for in silico-based virtual high throughput screening and molecular docking analysis of compounds as potential active site directed inhibitors of $Y 49 \beta$-lactamase}

The main purpose of this research was to find new 3 D-pharmacophores, i.e., non- $\beta$ lactam scaffolds that are potential active site, directed inhibitors of Y-49 $\beta$-lactamase. The first step in the development of a robust platform for molecular docking and in silico-based virtual high throughput screening of compounds requires finding a suitable X-Ray structure of the protein target of interest, preferably solved at high resolution $(<1.5 \AA) .{ }^{25,27}$ Given that all our in vitro enzymatic assays for screening of anti $\beta$-lactamase inhibitors were conducted with the Y-49 protein we began a bioinformatics search to find the structure of the protein template well-suited for in silico-based virtual screening involving large libraries of compounds. The Y-49 recombinant $\beta$-lactamase was identified previously as being the protein produced by a chromosomal gene coming from M. tuberculosis strain H37Rv, sequenced by the Sanger Center Cambridge, UK. ${ }^{31}$ The complete genome of H37Rv has 3999 coding genes. The list of expressed proteins in this genome acknowledged a class "A" $\beta$-lactamase protein having the following identifiers: accession number NP 216584.1 and locus tag Rv2068c (other names for Y-49 $\beta$-lactamase: blaC, blaA Rv2068c, MTCY49.07c (6)). The FASTA sequence of Y-49 $\beta$-lactamase was subjected to sequence similarity search using BLASTP and the protein hits with more than $99 \%$ sequence identity were searched against the Brookhaven RCSB PDB database and further screened for additional proteins with sequence and structural identity using the VAST $(+)$ algorithm and 
mMDB database (Figure 1) (Figure 2). This bioinformatics screening retrieved top ten hits having more than $99 \%$ sequence identity and less than $0.25 \AA$ RMSD for their structural similarity (Figure 2). One of the top ten hits, the 3M6B.pdb, revealed $100 \%$ sequence identity with Y-49 protein, except for a 42 -amino acid sequence located at $\mathrm{N}$-terminus of Y-49 that is used as the signal peptide for insertion in the membrane (highlighted in yellow in Figure 1B, (6)). The 100\% sequence identity of the two proteins justified the use of 3M6B.pdb as a target protein in the molecular docking experiments. Moreover, any of the top ten X-Ray reported structures shown in Figure 2 could be used for further structure-based drug discovery and virtual screening of active site binders for Y-49 $\beta$-lactamase because of their $99 \%$ and higher sequence identity with the original chosen target structure, 3M6B.pdb. Thus, these structures can be used in the future to validate the drug discovery screening platform optimized using 3M6B.pdb.

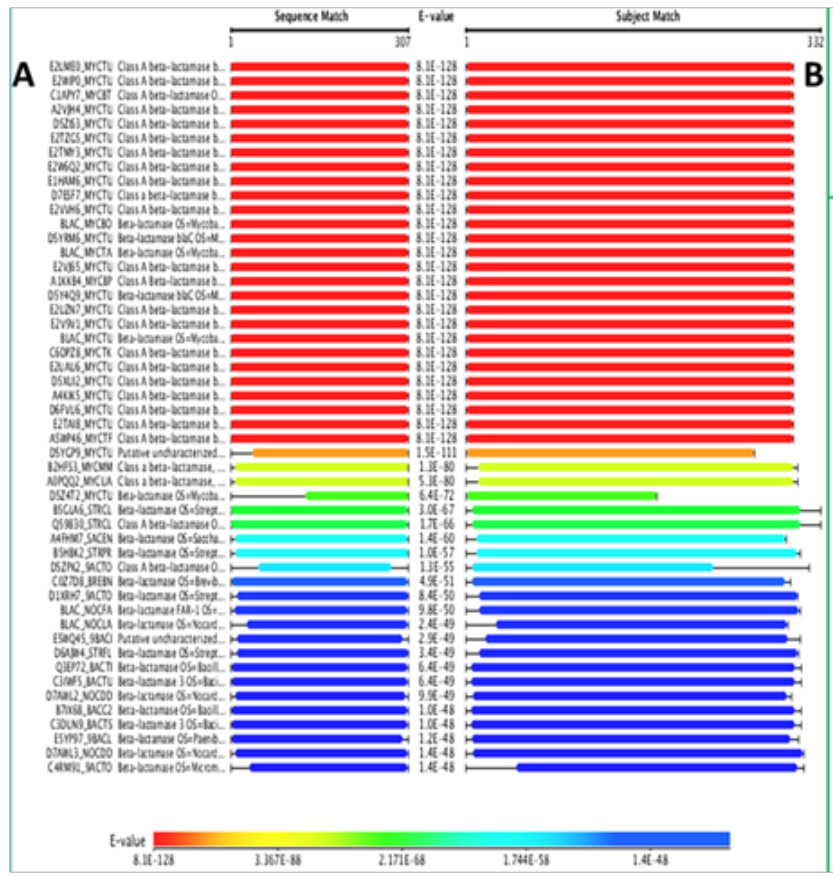

Amino Acid Sequence for Locus Tag Rv2068c H37Rv Strain

MRNRGFGRRELLVAMAMLVSVTGCARHASGARPASTTLPAGADLADRFAELERRYDA RLGVYVPATGTTAAIEYRADERFAFCSTFKAPLVAAVLHQNPLTHLDKLITYTSDDIRSIS PVAQQHVQTGMTIGQLCDAAIRYSDGTAANLLLADLGGPGGGTAAFTGYLRSLGDTVS RLDAEEPELNRDPPGDERDTTTPHAIALVLQQLVLGNALPPDKRALLTDWMARNTTGA KRIRAGFPADWKVIDKTGTGDYGRANDIAVVWSPTGVPYVVAVMSDRAGGGYDAEPR EALLAEAATCVAGVLA

\begin{tabular}{lllll}
\hline Score & Expect Method & Identifies & Positives & Gaps \\
\hline
\end{tabular}

536 bits(1382) 0.00 Compositional matixix adjust. 265/265(100\%) 265/265(100\%) 01265(0\%) Features:

Query 1 DLADRFAELERRYDARLGVYVPAGGTPAIEYRADEREARCSTFKAPLVAVLEQNPLYH 60

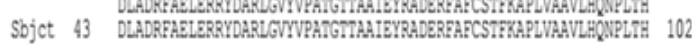

Qvery 61 LDKLITYTSDDIRSISPVAQQEVQIGMIGQLCDAIRYSDGIAANLLLADLGGPGGTA 120 LDKLITYTSDDIRSISPVAQQEVQIGYTIGQLCDAAIRYSDGTAANLLLADLGGPGGGT Sbjct 103 LDKLITYTSDDIRSISPVRQQEVVIGMTIGQLCDAAIRYSDGIAMLLLADLGGPGGGIA 162

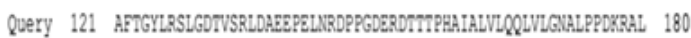
AFTGYRSLGOTVSRLDAEEPELIRDPPGDERDTTPPAATALVLOLULLGLLPPDKRAL Sbjct 163 AFTGYLSLGDTVSLDAEEPELNRDPPGDEROTTPPHATALVDOLIVLGNALPPDKRAL 222

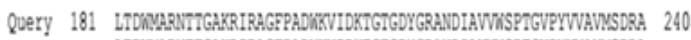
LTDWYARNTGAKRIRAG?PADKNVIDKTGIGDYGRANDAWWISTGVPYWAWYSDRA Sbjct 223 LTDRYARITGAKRIRAGPPADKVIDKTGIGDYGRADIAWNSPIGVYWWAWSDRA 282 Qvery 241 GGGYARPREALLAEATCVAGVIA 265 GGGYDAEREALLAEATCVAGILA Sbjct 283 GGGYDAEPREALLAEATCVAGVLA 307

Figure I The protein BLAST (BLASTp) search against the annotated NCBI protein database retrieved the Y-49 protein sequence as a class A $\beta$-lactamase (A) Further alignment of the Y-49 amino acid sequence with the amino acid sequence reported for 3M6B.pdb revealed $100 \%$ identity (with no gaps) spanning 265 amino acids in both proteins, except for a 42-amino acid sequence located at $\mathrm{N}$-terminus of $\mathrm{Y}-49$, used for insertion into membrane (6) (highlighted in yellow) (B). 3M6B.pdb was used as a target protein in the molecular docking experiments.

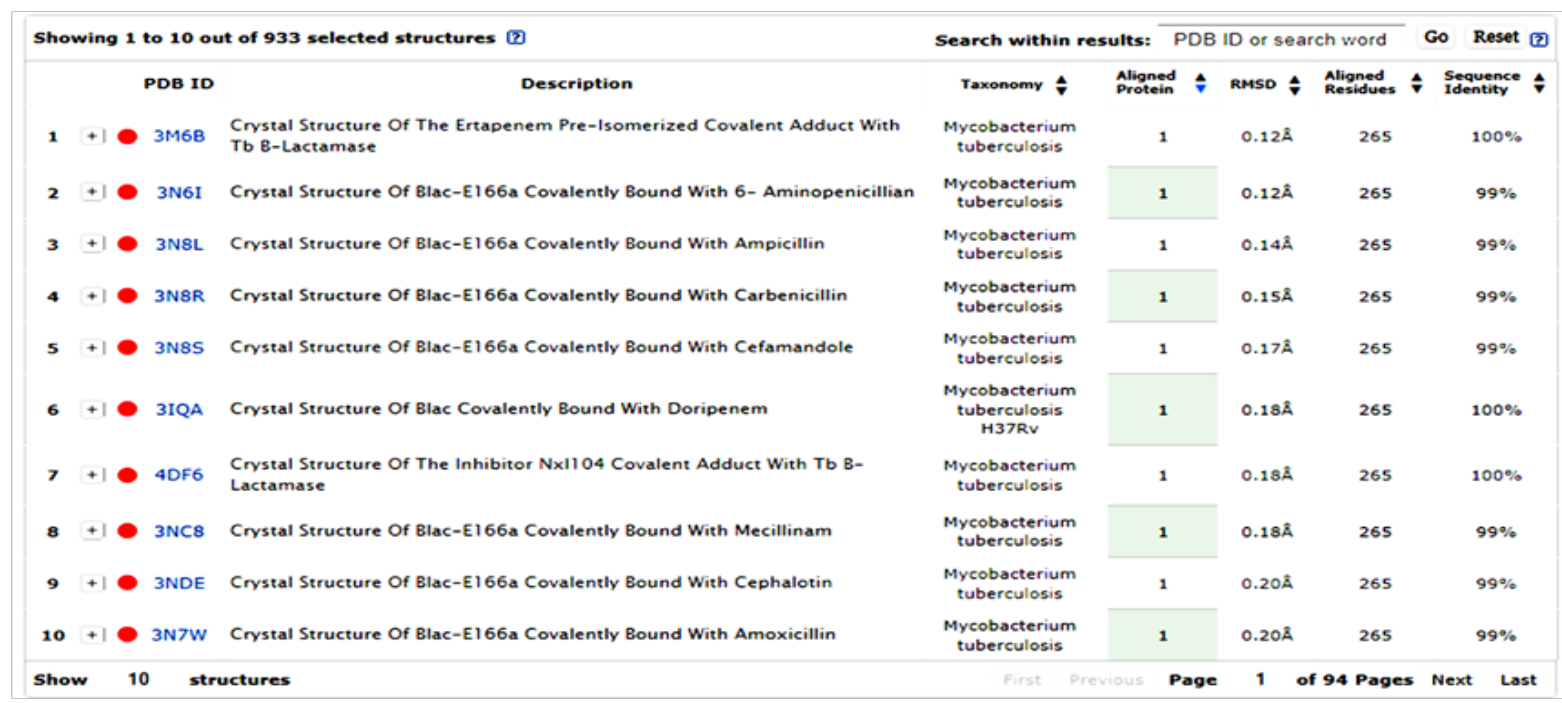

Figure 2 Top ten X-Ray structures retrieved by MMDB andVAST+ algorithm during searching for protein structures having more than $99 \%$ sequence identity with Y-49 protein. The potential proteins target suitable for performing the molecular docking and structure-based design were tracked for their structural similarities with the original chosen target 3M6B.pdb by employing the VAST+ analysis at MMDB and NCBI (25).

To determine whether compounds known as potential inhibitors of Y-49 $\beta$-lactamase could also exhibit inhibitory activity against other class A $\beta$-lactamases from other species of Mycobacterium or related genera, we performed an independent bioinformatics analysis on the conserved amino acids motifs within the active site of Y49, and 3M6B. Specifically, we used CLC bio Sequence Viewer (version 6.5, from 
QIAGEN) to perform the primary sequence alignments for selected $\beta$-lactamase proteins from class A that are important pharmacological targets. These included those from the Mycobacterium, Streptomyces, and Bacillus species: Mycobacterium kansasii, the causative agent of a pulmonary infection that resembles tuberculosis; M. marinum, which is the only Mycobacterium that is not aerobic; and M. ulcerans, the third most common mycobacterial pathogen in humans, after M. tuberculosis and M. leprae. ${ }^{33,34}$ In addition, we focused on finding the conserved amino acids motifs within the active site of Y49 protein in the $\beta$-lactamases from the Streptomyces species that are known for producing antibiotics. More than half of presently marketed antibiotics are produced by species belonging to the Streptomyces genus (A), such as cefoxitin, chloramphenicol, neomycin, streptomycin, tetracycline, and clavulanic acid. ${ }^{35}$ We also investigated the sequence similarities between Y49 protein and some beta lactamases from the Bacillus species known to produce a class A $\beta$-lactamase. ${ }^{36}$

The results from the primary sequence alignments are displayed in Figure 3 and highlight the presence of four distinct and highly conserved motifs within the active site of $3 \mathrm{M} 6 \mathrm{~B}$ and other $\beta$-lactamases from class A. Motif 1 consists of the sequence SXXK that contains the active site Ser84 residue. Motif 2 consists of the
SDN/G loop between helices $\alpha 4$ and $\alpha 5$. This Ser142 is involved in maintaining the functional position of the two domains and the protonation of the substrate's leaving group. ${ }^{6,36}$ Motif 3 consists of the acidic residue Glu182 having its side chain pointing into the substrate's binding cavity. Motif 4 consists of KTG sequence that is in the $\beta 3$ strand facing the SDN/G loop on the other side of the active site Ser84. The sequence logos for the identified conserved motifs in beta-lactamase proteins are presenting schematically the sequence conservation at each specific amino acid position; the height of symbols within each stack reflects the relative frequency of the corresponding amino acid (Figure 3). The four conserved motifs within the active site of Y-49 $\beta$-lactamase were further mapped in the 3D-structure of 3M6B using the molecular graphics software "UCSF Chimera" from the Resource for Biocomputing, Visualization, and Informatics at UCSF (https://www.cgl.ucsf.edu/chimera/) (Figure 4). Consequently, the bioinformatics analysis of primary and tertiary structures of Y-49 $\beta$-lactamase revealed conserved amino acids motifs spanning the active site of many $\beta$-lactamases from class A. This information was further used to perform structure-based design for discovery of non- $\beta$ lactam inhibitors of $\beta$-lactamase that could exhibit a wider antibacterial effect.

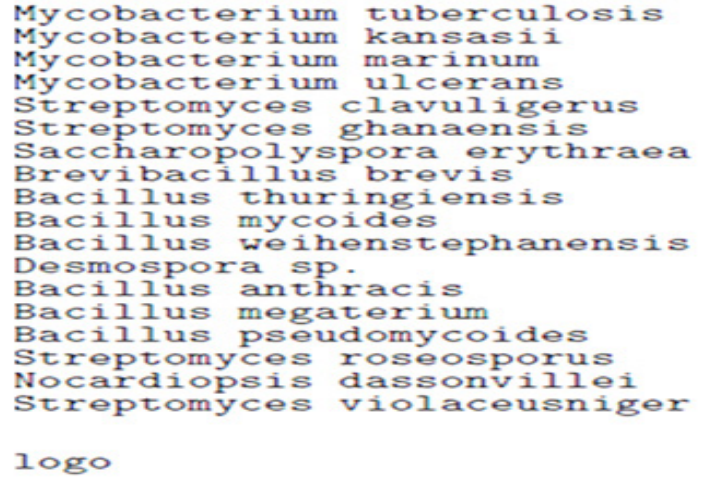

$10 g 0$
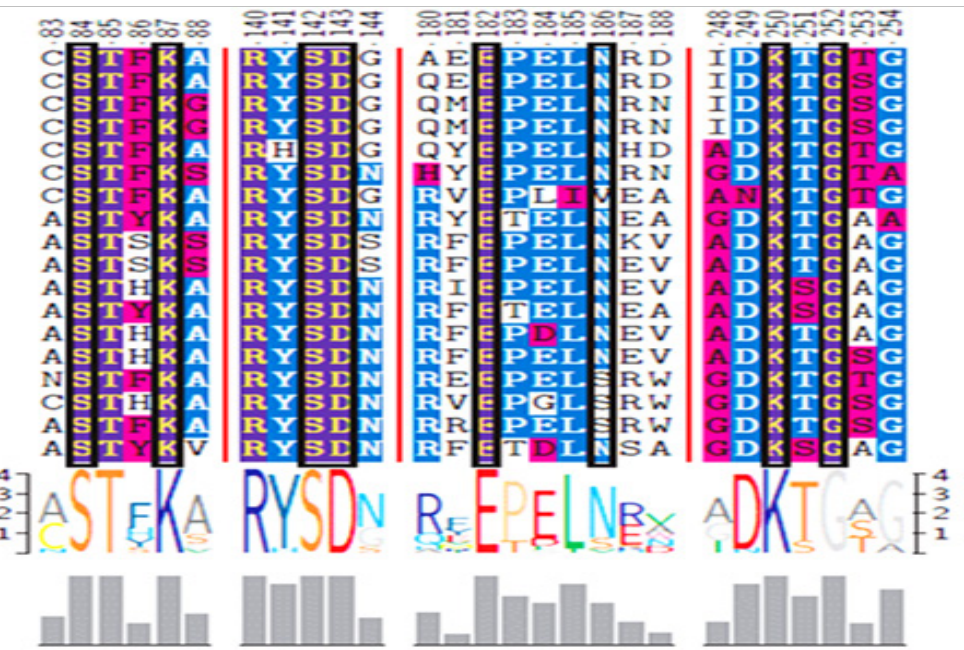

Figure 3 Analysis of the conserved motifs among the proteome of eighteen $\beta$-lactamases known as being of clinical interest. PSI-BLAST was used to identify homologues of 3M6B.pdb protein in the RefSeq and protein NCBI databases (figure I). The top eighteen proteins hits from PSI-BLAST output were exported and imported in the CLC Sequence Viewer where the alignment was performed to identify conserved sequence motifs. Sequence alignment displays the four highly distinct and conserved areas among the investigated $\beta$-lactamase family. The sequence logos of four conserved motifs in beta-lactamase proteins were displayed together with the position within the amino acid sequence. The overall height of each stack indicates the sequence conservation at that position, whereas the height of symbols within each stack reflects the relative frequency of the corresponding amino acid.

The 3M6B X-Ray structure allowed us to extract the coordinates of the ligand carbapenem (ertapenem (RG1)) co-crystalized as an adducted complex to the Ser84 within the active site of $3 \mathrm{M} 6 \mathrm{~B}$, and further input these data in the molecular docking platform. We next set up to develop and optimize the platform for performing the in silico-based virtual screening of large compound databases coupled with molecular docking using the online Pharmit server (http:// pharmit.csb.pitt.edu) (Figure 5). The MOE (molecular operating environment) (from CCG, Montreal, Canada) was used to extract the main pharmacophore features that define the interactions between the ligand ertapenem (RG1) and $\beta$-lactamase protein in the 3M6B.pdb structure. The MOE 2D-diagram option generated the 2D-view of the amino acid residues in close contact with the RG1 (ertapenem) ligand and displayed the main hydrogen bonds, salt bridges, hydrophobic and cation- $\pi$ interactions established by RG1 within the active site of 3M6B (Figure 5A). The original pose of RG1 was minimized in the active site of $\beta$-lactamase using the Amber12: EHT force field and the contribution of each protein residue-ligand atom interaction to the final reported free energy describing 3M6B/RG1 complex was calculated (Figure 5B). Remarkable contributions to the free energy of interaction between ertapenem and 3M6B $\beta$-lactamase are provided by the backbone amide nitrogen from Thr253 and Ser84. The oxygen atoms from some active site residues' side chains are further providing new hydrogen bonds donors (Glu182) or hydrogen bond acceptors (Ser142, Thr251) complementing the hydrogen bonding network that contribute more than " $-17.0 \mathrm{kcal} / \mathrm{mol}$ " to the relative free energy of interaction between RG1 and 3M6B. Selected water molecules are also contributing to more than " $-5.0 \mathrm{kcal} / \mathrm{mol}$," while the ionic interactions mediated by the side chains of Lys 250 and Arg236 contribute less than "- $2.0 \mathrm{kcal} / \mathrm{mol}$ " to the relative free energy of interaction. Notably, the functional groups in the RG1 ligand that establish the hydrogen bonding with the above-mentioned 
active site residues are carbonyl, free hydroxyl, carboxylate oxygen, and protonated amino groups. Hence, these chemical functional groups on the ligand can be beneficial in the first step for defining the next pharmacophore features. The map of the ligand-protein interactions in the original RG1/3M6B structure helped us to design the main steps for the platform enabling the on-line pharmacophore searching and screening of active site directed inhibitors for Y-49 $\beta$-lactamase (Figure 5B). The structure-based drug design (SBDD) approach required, as a first step, the interactive discovery of the best combination of pharmacophore features, i.e. hydrogen bonding acceptors and donors, hydrophobics, aromatics and electrostatics interactions describing the ligand-3M6B interactions within the active site, a task that was achieved with the help of Pharmit server (http:// pharmit.csb.pitt.edu). Virtual screening of large compound databases and SBDD using online Pharmit server (http://pharmit.csb.pitt.edu).

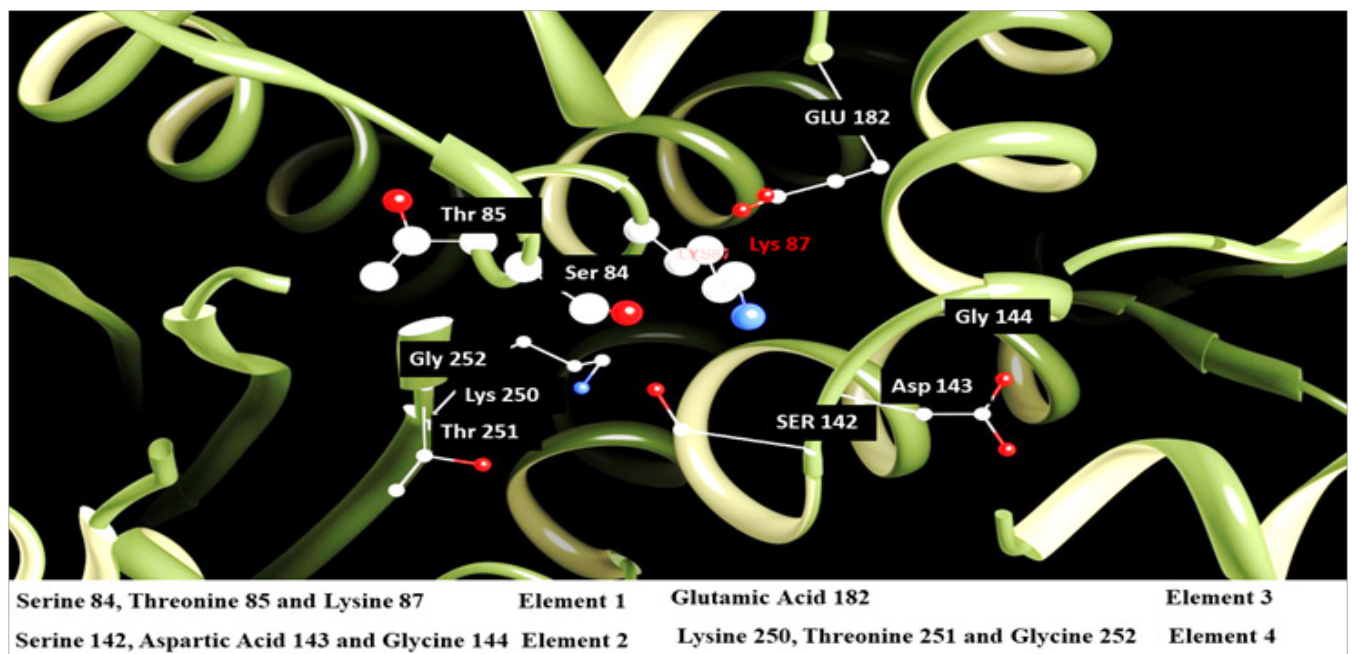

Figure 4 Three-dimensional view of 3M6B.pdb highlighting the amino acids identified by PSI-BLAST and CLC SequenceViewer to be part of the four conserved motifs (elements) in class A $\beta$-lactamase proteins. Molecular graphics images were produced by employing the UCSF Chimera package from the Resource for Biocomputing,Visualization, and Informatics at the University of California, San Francisco (https://www.cgl.ucsf.edu/chimera/).

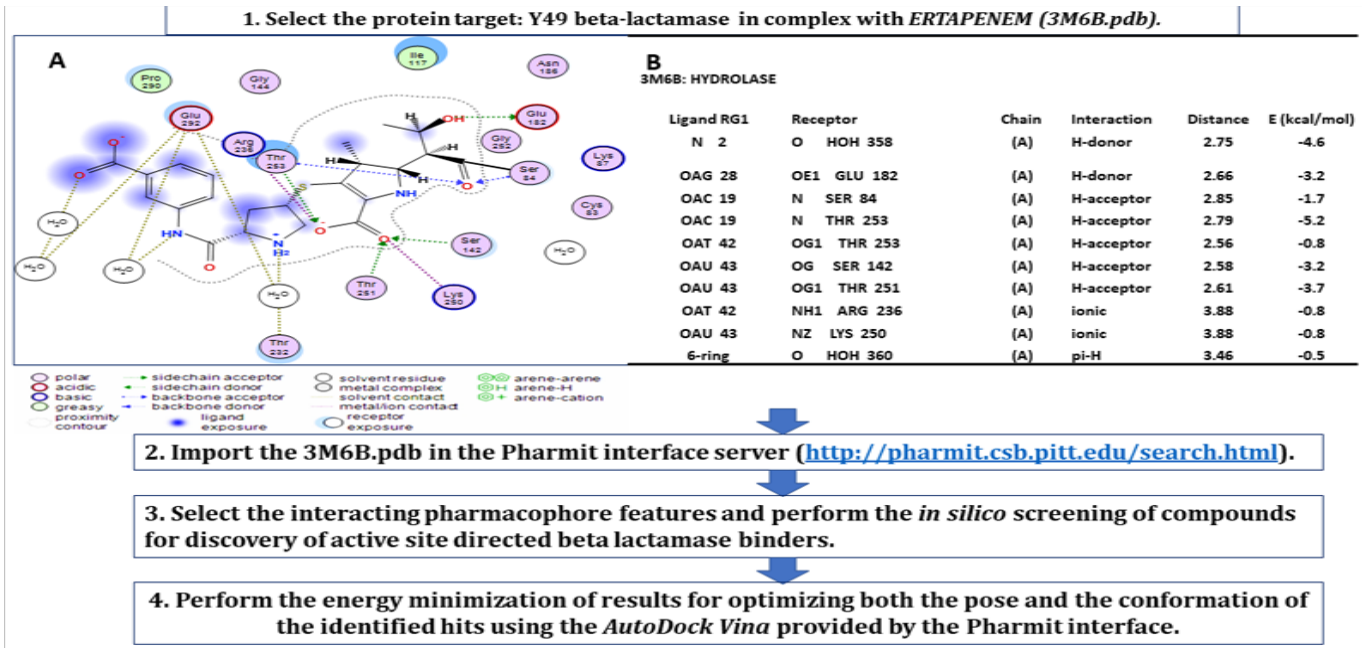

Figure 5 Workflow describing the procedure used to perform the in-silico screening of active site directed binders for Y-49 $\beta$-lactamase by employing pharmacophore searching and molecular docking with AutoDock Vina provided at the Pharmit server (http://pharmit.csb.pitt.edu). The MOE (Molecular Operating Environment) package was used to extract and visualize the main protein-ligand (carbapenem ertapenem (RGI)) interactions within $5 \AA$ from the ligand, in the target protein structure 3M6B.pdb (A). Details of the active site amino acid contribution to the free energy of interaction between the original ligand (RGI) and Y-49 $\beta$-lactamase are displayed as hydrogen bonding, electrostatic (ionic) and steric interactions with the original ligand from 3M6B.pdb (B).

In-silico mediated virtual screening of active site directed $\beta$-lactamase inhibitors was performed using the online comprehensive platform Pharmit. The best combination of pharmacophore features, i.e. hydrogen bond acceptor; hydrogen bond donor; hydrophobic; aromatics; and electrostatics were extracted from the original RG1/3M6B structure and accordingly, a predefined pharmacophore query was generated by Pharmit. For each selected pharmacophore features query we searched the compound libraries for matching compounds, including MolPort, CHEMBL21, ChemDiv, NCI Open Chemical Repository, PubMed and ZINC. The search was further restricted by applying a variety of filtering and ranking criteria, including those combining drug-like properties, observing Lipinski's "rule of five", as described in detail in the methods section..$^{22} \mathrm{~A}$ selected example of output from in silico-mediated virtual screening and molecular docking using AutoDock Vina at Pharmit server is reported in Figures $6 \& 7$. The SDF files generated from the virtual ligand screening in Pharmit were used as "chemical ligand libraries" to perform the docking in AutoDock Vina using the active site coordinates of 3M6B.pdb $\beta$-lactamase that in turn produced a ranked list of docked ligands from MolPort database (displayed in Figures 6 
\& 7). In addition to the list of compounds displayed in Figures 6 \& 7 other remarkable hits included: N2,N7-bis(2,3-dimethylphenyl)-9Hfluorene-2,7-disulfonamide (MolPort-000-687-571; characterized by an energy of interaction with $3 \mathrm{M} 6 \mathrm{~B}$ of " $-9.9 \mathrm{kcal} / \mathrm{mol}$ ") and N-benzyl2-9([2,5-dimethylphenyl]amino)-5-oxo-5H-(1,3,4) thiadiazolo (2,3-b) quinazoline-8-carboxamide (MolPort-007-764-070; characterized by an energy of interaction with $3 \mathrm{M} 6 \mathrm{~B}$ of " $-9.3 \mathrm{kcal}$ mol"). The compounds from sulfonamides and carboxamides series were previously shown by other research groups to be inhibitors of $\beta$-lactamase. ${ }^{37,38}$ Therefore, the original output from Pharmit validated our first platform designed to perform in silico screening of a large dataset of compounds by providing the above mentioned positive hits, i.e., known inhibitors for $\beta$-lactamase.

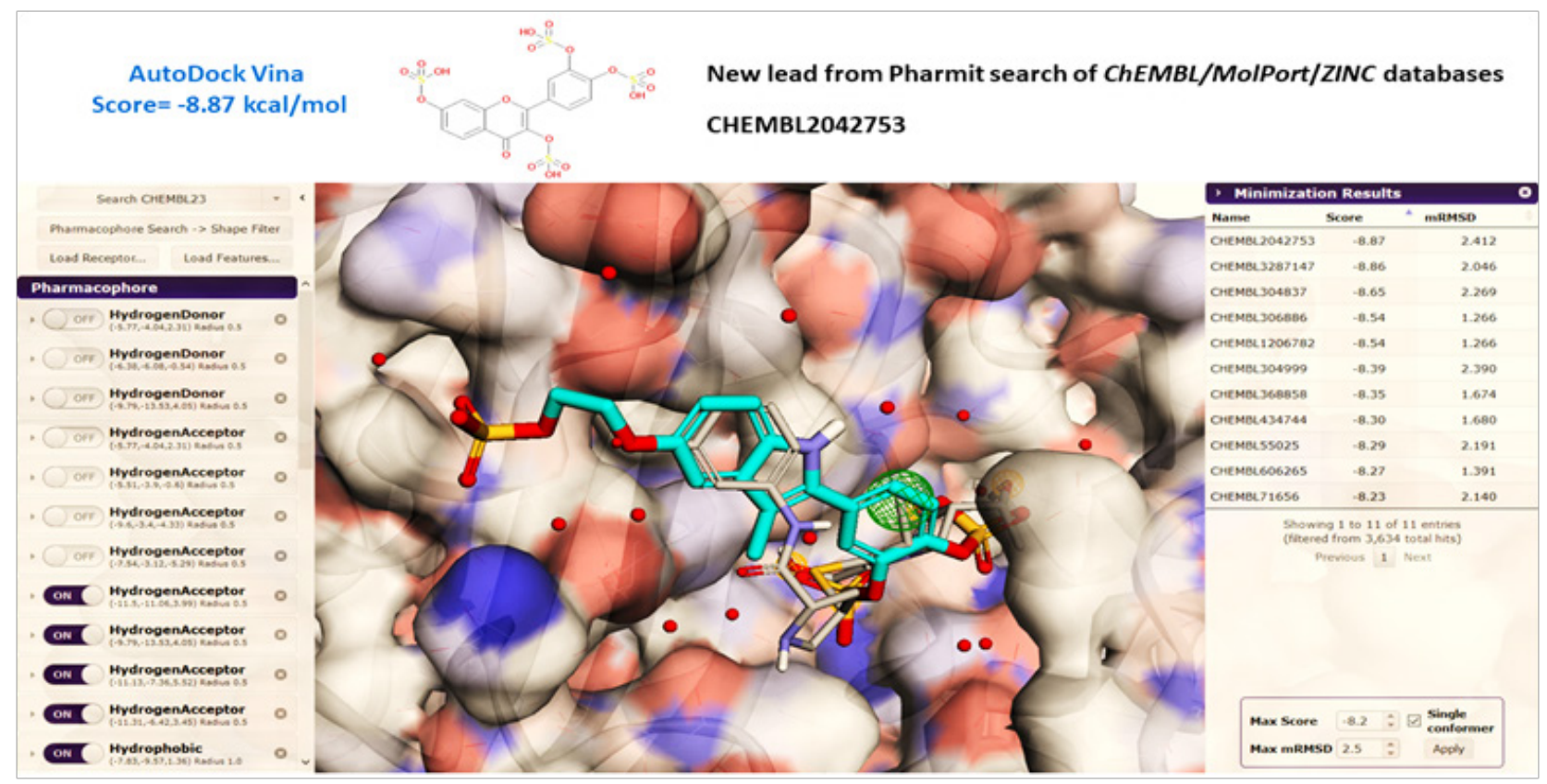

Figure 6 The pharmacophore searching page opened at the Pharmit server displaying the 3M6B.pdb structure of Y-49 $\square$-lactamase in complex with the Ser84 covalently adducted ertapenem (RGI), as primary query (CPK color). Each pharmacophore feature has a folding menu in the Pharmacophore panel (left) where its type, location, and radius, as well as number of atoms (for hydrophobic features) or directionality can be defined (20). Filters were set to reduce the number of hits by constraining the number of hits returned for a given conformer or molecule, or the overall number of hits (3-5 conformers/molecules). Selected pharmacophore features turned on (or off) are displayed on the left panel, and the table containing the compounds hits after one selected round of in silico screening are shown in the right panel, after energy minimization using the Autodock Vina provided by Pharmit. An example of a pharmacophore-aligned hit from the CHEMBIOL2I dataset for a query derived from PDB 3M6B is shown colored in blue turquoise while the original ligand is shown in CPK color (Right). The crystallographic water found in the active site of 3M6B structure are shown as red spheres. Pharmit allowed the water molecules to be chosen as part of the receptor active site.

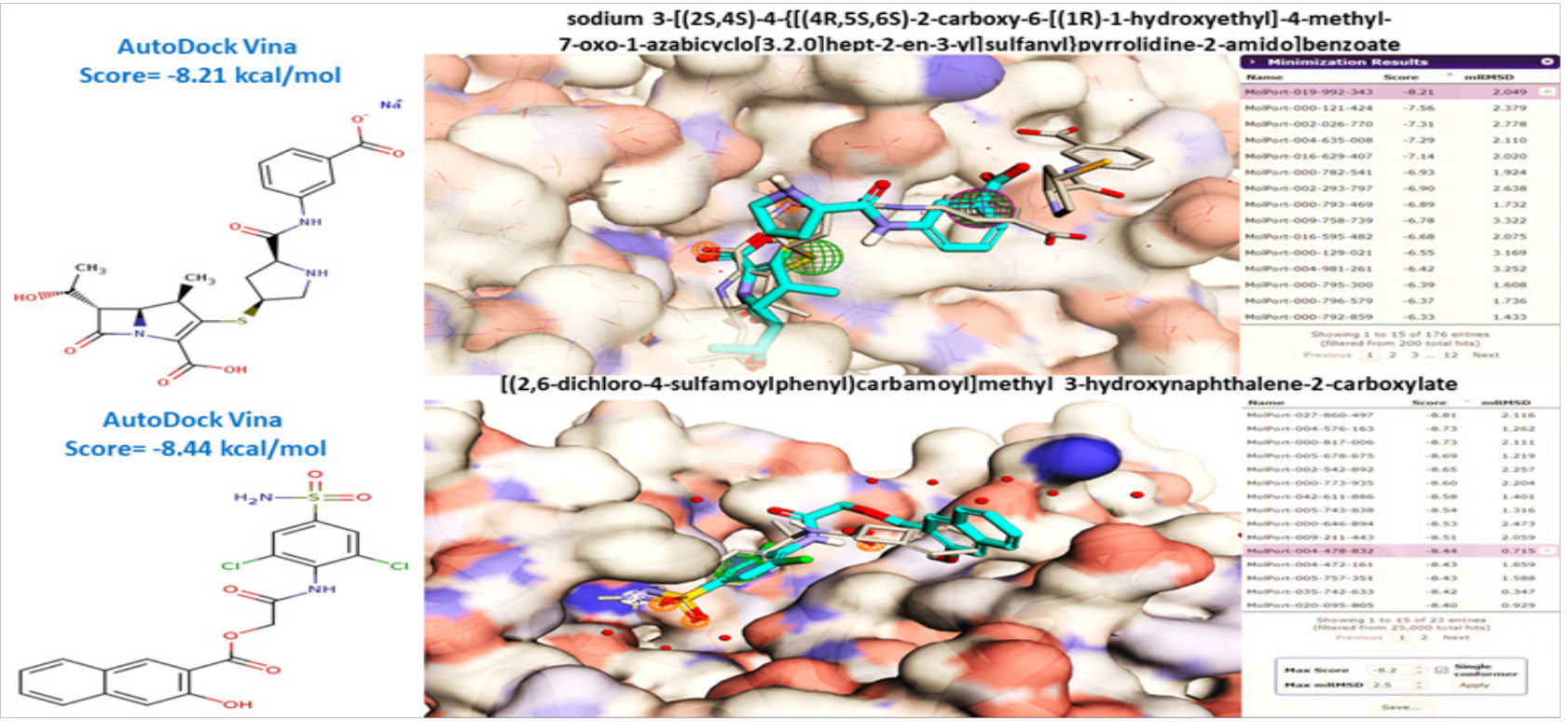

Figure 7 Independent examples of virtual screening and ranking of compounds using AutoDockVina provided by the Pharmit server (20). The pharmacophorealigned hits from the Molport dataset for a query derived from PDB 3M6B are shown colored in blue turquoise while the original ligand is shown in CPK color. The crystallographic water found in the active site of 3M6B structure are shown as red spheres. Pharmit allowed the water molecules to be chosen as part of the receptor active site.

Citation: Gonzalez J, Lendebol E, Shen A, et al. In silico-mediated virtual screening and molecular docking platforms for discovery of non $\beta$-lactam inhibitors of y-49 $\beta$-lactamase from Mycobacterium tuberculosis. MOJ Proteomics Bioinform. 2018;7(I):I2-24. DOI: 10.15406/mojpb.2018.07.00207 


\section{Discovery of novel 3D-pharmacophores and fragments from the family of diphenyl, aromatic ketones, hydroxy- phenyl glycine, and stilbenes as potential inhibitors of Y $49 \beta$-lactamase}

The virtual screening of large chemical libraries provided by Pharmit webserver enabled us to retrieve selected pharmacophore features, displayed for some hits in Figures $6 \& 7$. Specifically the multiple aromatic, phenolic groups substituted with hydroxyl, sulfonic groups and carbonylated compounds from the family of benzophenone and hydroxyl-benzophenone emerged as the top pharmacophore features candidates. We inquired further using a literature search at the PubChem database (https://pubchem.ncbi. nlm.nih.gov). This database contains all pharmacological agents described to have anti-bacterial and specifically anti-MTB activity, and encompassing the above-mentioned pharmacophore features. Not surprisingly we retrieved many lead compounds from the family of stilbenes and resveratrol. ${ }^{39,40}$ These findings prompted us to further analyze the possible relationship between the structure of diphenylhydroxylated compounds, e.g., stilbenes, and resveratrol, and their potential as $\beta$-lactamase inhibitors. Our motivation to investigate and determine whether there was a structure-activity relationship (SAR) between different stilbenes/resveratrol compounds and their anti- $\beta$-lactamase activity was also supported by all previous work reported on these pharmacological agents as inhibitors of some MTB strains growth. ${ }^{16-19,39,40}$ In 1944 Faulkner discovered the bactericidal properties of some stilbenes and diphenyl derivatives. ${ }^{19}$ The common structural feature for all tested compounds displaying bactericidal properties was the presence of one or more phenolic-type hydroxyl groups attached to the ring, and ethyl groups between the rings. ${ }^{16-19}$ Stilbenes are small molecules with molecular weights in the range of 200 to $300 \mathrm{~g} / \mathrm{mol}$. They are naturally-occurring plant secondary metabolites and are derived from the phenylpropanoid pathway. Stilbenes are structurally similar to estrogen and are produced in many unrelated plant species. ${ }^{16-18}$ When a plant is subjected to abiotic stress, such as drought, temperature, heavy metals, and salinity, the phenylpropanoid pathway is activated, and stilbenes are produced and secreted. Stilbenes are the plant's protective molecules; they are able to defend the plant from bacterial attack, as well as ultraviolet exposure. ${ }^{16-18}$ The most widely studied stilbene is resveratrol which has been shown to slow the progress of cancer. Moreover, it has been known for more than sixty years that resveratrol inhibits bacterial and fungal growth in vitro. ${ }^{16,17,39,40} \mathrm{In}$ the last decade it has been discovered that many stilbenes with a free hydroxyl groups were active against both bacteria and fungi with MICs in the range $25-100 \mu \mathrm{g} / \mathrm{ml}{ }^{16,39,40}$

However, no specific molecular targets were reported to be responsible for the observed antibacterial effects of resveratrol, or of other stilbenes with free hydroxyl groups. We first hypothesized that the structural features of such compounds would enable both good hydrophobic, van der Waals contacts and hydrogen bonding networks with selected amino acids from the Y49 $\beta$-lactamase. Indeed, when we analyzed the protein template $3 \mathrm{M} 6 \mathrm{~B}$ in complex with resveratrol we found the presence of selected hydrogen bonds between the ligand and active site residues. The "ligand map" generated by Molegro Viewer enabled the 2D-representation of all favorable hydrogen bonds, steric and electrostatic interactions established between the amino acids in the active site of $3 \mathrm{M} 6 \mathrm{~B}$ and resveratrol displayed in Figure 8 and summarized in Table 2. It can be seen from Table 2 that the amino acids from the active site of $\beta$-lactamase that established the hydrogen bonding network with the original ligand ertapenem, RG1, are also involved in making selected contribution to the relative free energy of interaction between the hydroxyl groups of resveratrol and 3M6B $\beta$-lactamase (i.e., Arg236, Glu182, Ser84, Ser142, Thr251 and Thr253). In addition, new active site residues show new contribution to the relative free energy of interaction of about "- $6.8 \mathrm{kcal} / \mathrm{mol}$." Specifically, the free hydroxyl from stilbene resveratrol make favorable hydrogen bonds with side chains of nitrogen and oxygen atoms from Lys87, Ser142, Asn186, Glu292, and with the backbone amide of Ile117 of $\beta$-lactamase (Figure 8). Consequently, we further hypothesized that the presence of free hydroxyl groups substituted to the benzene rings of other natural products derived from stilbenes, diphenyl compounds and other phenyl-hydroxyl containing fragments would enable similar hydrogen networks and thus provide relatively good binding affinity for the Y49 $\beta$-lactamase. To test our hypothesis, we selected some compounds from stilbenes, diphenyl derivatives (containing different double bonds positions, alkyl and substituted hydroxyl, amino and sulfonic functional groups), in addition to resveratrol, and performed the in vitro enzymatic assays to study their ability to inhibit the recombinant Y49 $\beta$-lactamase. In addition, we used other small organic molecules and fragments reported to display anti-bacterial activities and possessing phenyl, hydroxyl, and amino functionalities, e.g., D(-)-4-hydroxyphenylglycine. D(-)-4hydroxyphenylglycine is an important intermediate for the synthesis of penicillin, cephalosporin-type antibiotics including amoxycillin, celadroxil and cefatrizin and other semi-synthetic antibiotics, so its potential for inhibiting beta lactamase activity needed to be assessed. ${ }^{41}$ As can be seen from Figures $6 \& 7$, many of the selected small organic fragments and molecules are also constituents of the pharmacophore scaffolds displayed among the top drug candidates retrieved from the virtual screening of the Pharmit server. We next performed the inhibition assays using $24 \mu \mathrm{M}$ chromogenic nitrocefin substrate and $0.05-5 \mathrm{nM}$ enzyme concentration in a $200-250 \mu \mathrm{L}$ final volume of $25 \mathrm{mM}$ HEPES buffer, $\mathrm{pH}=7.4$. All kinetics assays tested different compounds at different concentrations, ranging $0.5-300 \mu \mathrm{M}$. The final $\mathrm{Ki}$ values were calculated from Morrison equation provided by the GraphPad Prism 7.0 (GraphPad Software) using initial rates approach described in detail in the methods. The Kis of selected compounds are reported in Table 1 together with the chemical ID structure (retrieved at PubChem database), and with the Autodock Vina predicted free energy of interaction with the target 3M6B. Table 1 also presents the experimentally-determined free energy of interaction between different compounds and $\beta$-lactamase for comparison with the theoretical prediction. It was observed that Autodock Vina predicted with more than $80 \%$ accuracy the free energy of interaction between some of the tested small organic molecules and their target protein (in this research $3 \mathrm{M} 6 \mathrm{~B}$ ), as was the case with resveratrol and most of the tested stilbenes reported in Table 1. However, Table 1 also displays that Autodock Vina failed to predict with similar accuracy the affinity for some small organic fragments, such as benzylamine, hydroxybenzylamine, hydroxyglycine and 2-aminohexanol.

Selected kinetics traces for the assays performed with the fragment inhibitors benzophenone and 2-aminohexanol are displayed in Figure 9. It can be seen from Table 1 that benzophenone and dibenzylsulfone showed relative good inhibition against recombinant $Y 49 \beta$-lactamase ( $\mathrm{K}_{i}$ for benzophenone $=25.42 \mu \mathrm{M}$ and $\mathrm{K}_{i}$ for dibenzylsulfone $=16.56 \mu \mathrm{M}$ ). We hypothesized that both compounds, having good electrophilic functional groups, could eventually be subjected to the interaction with the nucleophilic $\gamma \mathrm{O}$ of the active site Ser84. The nucleophilic attack of gamma oxygen $(\gamma \mathrm{O})$ from Ser84 to the carbonyl carbon of benzophenone and the sulphur center of dibenzylsulfone $(+2$ oxidation state) should mimic the same interaction with a $\beta$-lactam antibiotic. This hypothesis was indirectly confirmed by the fact that 
the benzophenone imine and diphenyl amine had no inhibitory effect (not shown in Table 1). We explained their lack of activity being due to presence of the imine $\mathrm{C}=\mathrm{N}$ bond that is less polar than $\mathrm{C}=\mathrm{O}$ bond, giving the nucleophilic of $\gamma \mathrm{O}$ Ser84 no attack site, and leaving the lone pair electrons of nitrogen in diphenylamine involved in $\pi-\pi$ interactions with the phenyl groups. This resonance effect would make it less susceptible to nucleophilic attack. The requirement for having electrophilic centers and free hydroxyl groups in the structure of the pharmacophore exhibiting potential inhibitory activity against
Y49 $\beta$-lactamase was further validated by the weak inhibition obtained with the diphenyl fragment that is non-polar, and that does not have electrophilic centers (relative high $\mathrm{Ki}=123 \mu \mathrm{M}$ ). In addition to the requirement of having diphenyl and multiple free hydroxyl groups, the SAR presented in Table 1 shows that other fragments and 3D-chemical scaffolds could be further explored as potential anti Y49 $\beta$-lactamase inhibitors; remarkably, the scaffold provided by $\mathrm{D}(-)-$ 4-Hydroxyphenylglycine is a new fragment lead with an inhibitory constant $\mathrm{Ki}=5 \mu \mathrm{M}$, being followed by dibenzylsulfone with $\mathrm{Ki}=17 \mu \mathrm{M}$.

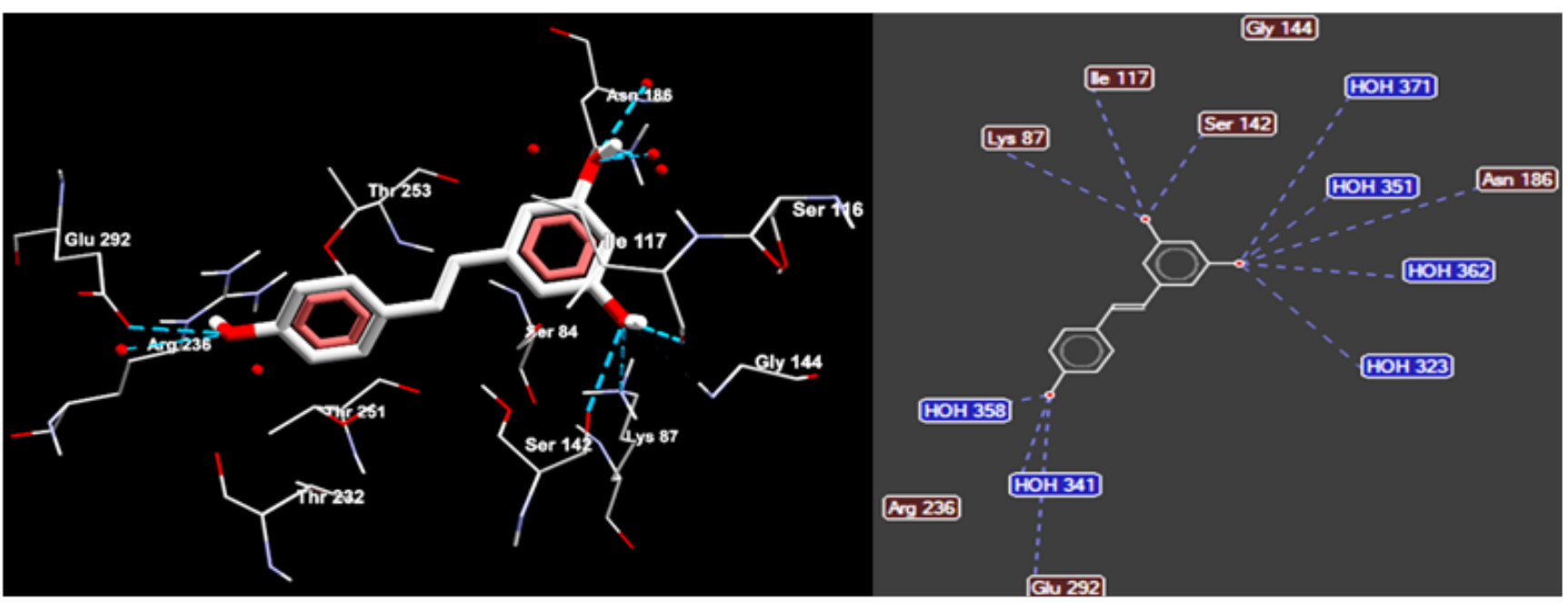

Figure 8 MolegroViewer enabled the three-dimensional display of the structural details describing the interaction between the best docked pose of resveratrol (CPK colored) and the active site residues of 3M6B $\beta$-lactamase (left panel). AutoDock Vina was used to perform the rigid docking which predicted a free energy of about "-7.0 kcal/mol" characterizing the interaction between the resveratrol and $\beta$-lactamase. The hydrogen bonds established between the hydroxyl oxygen groups from resveratrol and the selected amino acids and water molecules in the active site of $\beta$-lactamase are displayed as dotted blue lines (right panel).

Table I Structure activity relationship (SAR) for the fragment pharmacophores, small organic molecules and selected non-beta lactam scaffolds originally discovered from the in-silico screening of selected chemical scaffolds provided by the Pubchem open chemistry database (https://pubchem.ncbi.nlm.nih.gov), and the pharmacophore features screening provided by the Pharmit web server (as shown in figures 6 and 7). The fragment pharmacophores and the compounds were originally tested for their ability to inhibit the $Y-49$ beta-lactamase in an in vitro enzymatic assay using the chromogenic nitrocefin substrate.Virtual screening and molecular docking using AutoDockVina provided the predicted free energy for the interaction between the ligand and protein target $3 \mathrm{M} 6 \mathrm{~B}$ (i.e. the affinity score, in " $\mathrm{kcal} / \mathrm{mol}$ "). The experimental free energy of interaction (in $\mathrm{kcal} / \mathrm{mol}$ ) was calculated using the formula: $\Delta \mathrm{G}=-\mathrm{RT} / \mathrm{nK}$ eq $\left(\right.$ where $\mathrm{R}=-0.00 \mathrm{I} 987 \mathrm{kcal} . \mathrm{K}^{-1}$. $\mathrm{mol}^{-1} ; \mathrm{T}=310 \mathrm{~K}\left(37^{\circ} \mathrm{C}\right)$; and $\left.\mathrm{Keq}=\mathrm{I} / \mathrm{Ki}\right)$.

\section{Compound Name}

Pubchem ID(CID)/Structure

$\begin{array}{lll}\text { Autodock Vina } & \text { Experimental Ki } & \begin{array}{l}\text { Experimental } \\ \text { Predicted Affinity }\end{array} \\ \text { Score (kcal/mol) } & (\mu \mathrm{M}) & \text { Affinity (kcal/mol) }\end{array}$

Benzylamine

CID 7504

2-hydroxybenzylamine

CID 70267

D (-)-4-Hydroxyphenylglycine

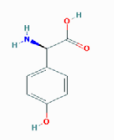

CID 89853

$-5.7$

$5 \pm 2$

$-7.20$

$-6.3$

Benzophenone

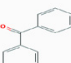

CID 3102
$25 \pm 2$

$-6.52$ 
Table Continued....

\begin{tabular}{|c|c|c|c|c|}
\hline Compound Name & Pubchem ID(CID)/Structure & $\begin{array}{l}\text { Autodock Vina } \\
\text { Predicted Affinity } \\
\text { Score (kcal/mol) }\end{array}$ & $\begin{array}{l}\text { Experimental Ki } \\
(\mu \mathrm{M})\end{array}$ & $\begin{array}{l}\text { Experimental } \\
\text { Affinity (kcal/mol) }\end{array}$ \\
\hline Dibenzylsulfone & CID 69282 & -6.2 & $17 \pm 3$ & -6.70 \\
\hline dl-2-Amino-I-hexanol & CID 249006 & -4.2 & $19 \pm 4$ & -6.69 \\
\hline 2,2-Dihydroxybenzophenor & CID 70038 & -6.5 & $40 \pm 5$ & -6.24 \\
\hline 4-Hydroxystilbene & CID 5284650 & -6.1 & $|4| \pm 12$ & -5.40 \\
\hline \multicolumn{5}{|l|}{$\begin{array}{l}\text { 4,4'-Diaminostilbene-2,2'- } \\
\text { disulphonic acid }\end{array}$} \\
\hline \multicolumn{5}{|l|}{ 4-Nitrostilbene } \\
\hline & CID 9800I & -6.7 & $56 \pm 8$ & -6.03 \\
\hline \multicolumn{5}{|l|}{ Diethylstilbestrol } \\
\hline & CID 448537 & -6.5 & $32 \pm 5$ & -6.40 \\
\hline \multicolumn{5}{|l|}{ Resveratrol } \\
\hline 3,5,4'-(Trihydroxystilbene) & CID $445 I 54$ & -6.8 & $14 \pm 4$ & -6.9 \\
\hline
\end{tabular}

We finally were able to define a 3D pharmacophore featuring the key structural requirements characterizing the interaction between the tested stilbenes, aromatics ketones and diphenyl compounds with the amino acids residues within the active site of Y49 $\beta$-lactamase: 1 ) interaction of any electrophilic center with either the active-site Ser84 or Lys87 to putatively form a hydrogen bond within the active site cavity, and 2) correct orientation of the $\mathrm{OH}$ (or other amino, carboxyl, carboxyamide, sulfonamide) groups to elicit hydrogen bonding interactions with either the main chain carbonyl oxygen of Ser142 and Gly252 or the amino group from the side chain of Asn186. Moreover, the molecular docking experiments predicted that the establishment of additional hydrogen bonding networks involving the residues Asn186, Glu292, Thr251, Thr253, Arg 236, and Lys250 would increase their

relative binding affinity for Y49 $\beta$-lactamase (see Table 2 for the case of resveratrol).

The SAR presented in Table 1 are, to our knowledge, the first to show one of the possible molecular mechanisms of action for some stilbenes, resveratrol and diphenyl derivatives which could explain their already reported antibacterial activities. ${ }^{16-19,39,40}$ Consequently, the development of stilbenes derivatives and other diphenyl compounds with selected electrophilic functionalities could provide an independent approach to develop non- $\beta$-lactam compounds inhibitors of $\beta$-lactamases from class $A$, and complement other approaches used today to overcome the Mycobacterium tuberculosis resistance to antibiotics. 

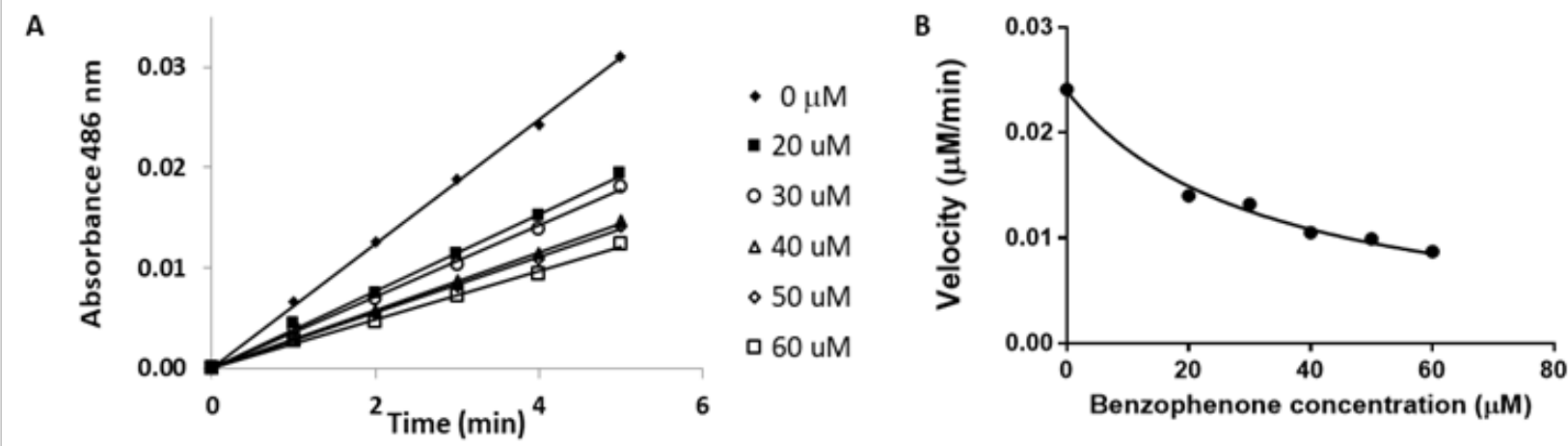

Figure 9 Selected kinetics traces run at different concentrations for the tested chemical fragment inhibitor benzophenone. Initial velocity experiments were performed in $25 \mathrm{mM}$ HEPES buffer, $\mathrm{pH} 7.4$, in presence of chromogenic nitrocefin substrate $(24 \mu \mathrm{M})$ and recombinant $Y-49 \beta$-lactamase $(0.5-5 \mathrm{nM})$ for the selected time points and at different concentrations of compound (20-70 $\mu \mathrm{M})$. The reaction initial velocity was calculated using the reported extinction coefficient for nitrocefin at $486 \mathrm{~nm}(\varepsilon=20,500 \mathrm{M}-\mathrm{I} \mathrm{cm}-\mathrm{I})$, and plotted as a function of each inhibitor concentration: benzophenone (B). The final Ki values were calculated from Morrison equation provided by the GraphPad Prism 7.0 (GraphPad Software), and independently from a plot of Ki=[[I]-[E](Vr-I)]/(I/Vr-I); where $\mathrm{Vr}=\mathrm{V} / \mathrm{V} 0$ is the relative velocity, $[\mathrm{l}]=$ concentration of inhibitor (in $\mu \mathrm{M}$ ) and $[\mathrm{E}]=$ concentration of enzyme (in $\mu \mathrm{M})(32)$.

Table 2 EPair energy terms, expressed as MolDock scoring functions generated by Molegro Dock software for one selected lead compound, resveratrol, using the virtual screening protocol at Pharmit server. The presented MolDock scoring function consists of mainly the pairwise (PLP) steric and hydrogen bonding energy of interaction between the resveratrol and the amino acid residues from the active site of Y-49 $\beta$-lactamase target 3M6B [chain A]. The compound was predicted to have less than "-7.0kcal/mol" relative free energy of interaction with $Y-49$-lactamase based on the scoring function generated by the molecular docking module AutoDockVina provided by the Pharmit server.The pose predicted to have the best free energy of interaction with $\beta$-lactamase in the context of 3M6B structure is displayed in Figures 8

\begin{tabular}{|c|c|c|c|}
\hline Target atoms: molecule (Resveratrol) & Residue & ID & EPair \\
\hline $3 M 6 B[A]$ & Arg & 236 & -5.98847 \\
\hline $3 \mathrm{M} 6 \mathrm{~B}[\mathrm{~A}]$ & Asn & 186 & -4.07778 \\
\hline $3 \mathrm{M} 6 \mathrm{~B}[\mathrm{~A}]$ & Asp & 143 & $-1.0807 \mid$ \\
\hline $3 \mathrm{M} 6 \mathrm{~B}[\mathrm{~A}]$ & Glu & 182 & -4.7988 \\
\hline $3 \mathrm{M} 6 \mathrm{~B}[\mathrm{~A}]$ & Glu & 292 & -3.46133 \\
\hline $3 \mathrm{M} 6 \mathrm{~B}[\mathrm{~A}]$ & Gly & 144 & -1.82584 \\
\hline $3 M 6 B[A]$ & Gly & 252 & -1.44514 \\
\hline $3 \mathrm{M} 6 \mathrm{~B}[\mathrm{~A}]$ & Gly & 254 & -0.30411 \\
\hline $3 \mathrm{M} 6 \mathrm{~B}[\mathrm{~A}]$ & lle & 117 & -19.8017 \\
\hline $3 \mathrm{M} 6 \mathrm{~B}[\mathrm{~A}]$ & Lys & 87 & -3.42984 \\
\hline $3 \mathrm{M} 6 \mathrm{~B}[\mathrm{~A}]$ & Ser & 84 & -4.6 \\
\hline $3 M 6 B[A]$ & Ser & 116 & -2.35401 \\
\hline $3 \mathrm{M} 6 \mathrm{~B}[\mathrm{~A}]$ & Ser & 142 & -8.47773 \\
\hline $3 \mathrm{M} 6 \mathrm{~B}[\mathrm{~A}]$ & Thr & 232 & -7.33538 \\
\hline $3 \mathrm{M} 6 \mathrm{~B}[\mathrm{~A}]$ & Thr & 251 & -3.87346 \\
\hline $3 \mathrm{M} 6 \mathrm{~B}[\mathrm{~A}]$ & Thr & 253 & -16.692 \\
\hline $\mathrm{HOH} 2 \mathrm{I}$ & (water) & 11 & -1.90048 \\
\hline $\mathrm{HOH} 323$ & (water) & 37 & -3.30957 \\
\hline $\mathrm{HOH} 34 \mathrm{I}$ & (water) & 57 & -1.97339 \\
\hline $\mathrm{HOH} 35 \mathrm{I}$ & (water) & 68 & -3.40334 \\
\hline $\mathrm{HOH} 358$ & (water) & 75 & 25.0547 \\
\hline $\mathrm{HOH} 362$ & (water) & 80 & 11.8365 \\
\hline $\mathrm{HOH} 37 \mathrm{I}$ & (water) & 90 & 9.83372 \\
\hline HOH 397 & (water) & 118 & -2.96275 \\
\hline $\mathrm{HOH} 426$ & (water) & $|5|$ & -1.49992 \\
\hline $\mathrm{HOH} 475$ & (water) & 200 & -0.3066 \\
\hline
\end{tabular}

Citation: Gonzalez J, Lendebol E, Shen A, et al. In silico-mediated virtual screening and molecular docking platforms for discovery of non $\beta$-lactam inhibitors of y-49 $\beta$-lactamase from Mycobacterium tuberculosis. MOJ Proteomics Bioinform. 20 I8;7(I):I2-24. DOI: 10.15406/mojpb.20I8.07.00207 


\section{Conclusion}

In conclusion, the research presented herein demonstrated the successful development of an in silico-based virtual screening coupled with molecular docking platform that enabled the discovery of novel, non- $\beta$-lactam $\beta$-lactamase inhibitors of Y-49 $\beta$-lactamase from Mycobacterium tuberculosis. This was accomplished by employing the 3D-pharamacophore-based virtual screening provided by the on-line server Pharmit (pharmit.csb.pitt.edu), coupled with multiple rounds of in vitro screening platform using kinetics of $\beta$-lactamase inhibition with nitrocefin substrate. Specifically, the in-silico virtual screening of large chemical datasets provided by Pharmit (such as Molport, CHEMBL21, ChemDiv, NCI Open Chemical Repository, PubMed and ZINC database) allowed us to improve the structure-based drug design and discovery of novel inhibitors of Y-49 $\beta$-lactamase. Notably, new 3D-pharmacophore and fragments from the family of diphenyl, aromatic ketone, hydroxy-phenyl glycine, stilbenes and resveratrol emerged as potential inhibitors of Y-49 $\beta$-lactamase and exhibited inhibitory constants, $\mathrm{Ki}$, in the low $5 \mathrm{uM}-200 \mathrm{uM}$ in an in vitro enzymatic assay with nitrocefin substrate. To our knowledge, this new SBDD and SAR on stilbenes, resveratrol and hydroxylated diphenyl compounds is the first to show that their inhibitory activity against Y49 $\beta$-lactamase, possibly revealing the molecular mechanisms that explain their already reported antibacterial activities. These newlydiscovered pharmacophores features are extending the chemical space for discovery of novel and potent non $\beta$-lactam inhibitors of $\beta$-lactamases.

\section{Acknowledgements}

Support for this project was provided by the CUNY Research Scholars Program, which is funded by The City University of New York and the New York City Mayor's Office.

\section{Conflict of interest}

The author declares no conflict of interest.

\section{References}

1. Frere JM. Beta-lactamases and bacterial resistance to antibiotics. Mol Microbiol. 1995;16(3):385-395.

2. Drawz SM, Bonomo RA. Three decades of beta-lactamase inhibitors. Clin Microbiol Rev. 2010;23(1):160-201.

3. Bush K, Jacoby GA. Updated functional classification of $\beta$-lactamses. Antimicrob Agents Chemother. 2010;54(3):969-976.

4. Helfand MS, Bonomo RA. $\beta$-Lactamases: A Survey of Protein Diversity. Curr Drug Targets: Infect Disord. 2003;3(1):9-23.

5. Hugonnet JE, Tremblay LW, Boshoff HI, et al. Meropenem-clavulanate is effective against extensively drug-resistant Mycobacterium tuberculosis. Science. 2009;323(5918):1215-1218.

6. Hazra S, Kurz SG, Wolff K, et al. Kinetic and Structural Characterization of the Interaction of 6-Methylidene Penem 2 with the $\beta$-Lactamase from Mycobacterium tuberculosis. Biochemistry. 2015;54(36):5657-5664.

7. Zhanel GG, Lawrence CK, Adam H, et. al. Imipenem-Relebactam and Meropenem-Vaborbactam: Two Novel Carbapenem- $\beta$-Lactamase Inhibitor Combinations. Drugs. 2017;78(1):65-98.

8. Zhanel GG, Lawson CD, Adam H, et al. Ceftazidime-avibactam: a novel cephalosporin/ $\beta$-lactamase inhibitor combination. Drugs. 2013;73(2):159-177.

9. Hecker SJ, Reddy KR, Totrov M, et al. Discovery of a Cyclic Boronic Acid $\beta$-Lactamase Inhibitor (RPX7009) with Utility vs Class A Serine Carbapenemases. J Med Chem. 2015;58(9):3682-3692.
10. Sgrignani J, De Luca F, Torosyan H, et al. Structure-based approach for identification of novel phenylboronic acids as serine- $\beta$-lactamase inhibitors. J Comput Aided Mol Des. 2016;30(10):851-861.

11. Mandal SM, Migliolo L, Silva ON, et al. Controlling resistant bacteria with a novel class of $\beta$-lactamase inhibitor peptides:from rational design to in vivo analyses. Sci Rep. 2014;4:6015.

12. Drawz SM. New $\beta$-lactamase inhibitors: a therapeutic renaissance in an "MDR world. Antimicrob Agents Chemother. 2014;58(4):1835-1846.

13. Lohan S, Bisht GS. Recent approaches in design of peptidomimetics for antimicrobial drug discovery research. Mini Rev Med Chem. 2013;13(7):1073-1088

14. Méndez-Samperio P. Peptidomimetics as a new generation of antimicrobial agents: current progress. Infect Drug Resist. 2014;7:229 237.

15. Klingler FM, Wichelhaus TA, Frank D, et al. Approved drugs containing thiols as inhibitors of metallo- $\beta$-lactamases: strategy to combat multidrug-resistant bacteria. J Med Chem. 2015;58(8):3626-3630.

16. Aslam SN, Stevenson PC, Kokubun T, et al. Antibacterial and antifungal activity of cicerfuran and related 2-arylbenzofurans and stilbenes. Microbiol Res. 2009;164(2):191-165.

17. Lee WX, Basri DF, Ghazali AR. Bactericidal Effect of Pterostilbene Alone and in Combination with Gentamicin against Human Pathogenic Bacteria. Molecules. 2017:22(3).

18. Välimaa AL, Honkalampi-Hämäläinen $\mathrm{U}$, Pietarinen $\mathrm{S}$, et al Antimicrobial and cytotoxic knotwood extracts and related pure compounds and their effects on food-associated microorganisms. Int $J$ Food. 2007;115(2):235-243.

19. Faulkner GH. The antibacterial action of some biphenyl derivatives Biochem J. 1944;38(5):370-372.

20. Sunseri J, Koes DR. Pharmit: interactive exploration of chemical space. Nucleic Acids Res. 2016;44(W1):W442-W448.

21. Koes DR, Camacho CJ. ZINC Pharmer: pharmacophore search of the ZINC database. Nucleic Acids Res. 2012;40(Web Server issue):W409W414.

22. Lipinski CA. Lead- and drug-like compounds: the rule-of-five revolution. Drug Discovery Today Technol. 2004;1(4):337-341.

23. Helgren TR, Hagen TJ. Demonstration of AutoDock as an Educational Tool for Drug Discovery. J Chem Educ. 2017;94(3):345-349.

24. Johnson M, Zaretskaya I, Raytselis Y, et al. NCBI BLAST: a better web interface. Nucleic Acids Res. 2008;36(Web Server issue):W5-W9.

25. Madej T, Lanczycki CJ, Zhang D, et al. MMDB and VAST+: tracking structural similarities between macromolecular complexes. Nucleic Acids Res. 2014;42(Database issue):D297-D303.

26. Tremblay LW, Fan F, Blanchard JS. Biochemical and structural characterization of Mycobacterium tuberculosis beta-lactamase with the carbapenems ertapenem and doripenem. Biochemistry. 2010;49(17):3766-3773.

27. Corbeil CR, Williams CI, Labute P. Variability in Docking Success Rates Due to Dataset Preparation. J Comp Aided Mol Des. 2012;26(6):775786.

28. Labute P. Protonate3D: Assignment of Ionization States and Hydrogen Coordinates to Macromolecular Structures. Proteins. 2008;75(1):187205

29. Clark AM, Labute P. 2D Depiction of Protein-Ligand Complexes. $J$ Chem Inf Model. 2007;47(5):1933-1944.

30. Feng DF, Doolittle RF. Progressive sequence alignment as a prerequisite to correct phylogenetic trees. J Mol Evol. 1987;25(4):351-360. 
31. Voladri RK, Lakey DL, Hennigan SH, et al. Recombinant expression and characterization of the major beta-lactamase of Mycobacterium tuberculosis. Antimicrob Agents Chemother. 1998;42(6):1375-1381.

32. Murphy DJ. Determination of accurate KI values for tight-binding enzyme inhibitors: an in-silico study of experimental error and assay design. Anal Biochem. 2004;327(1):61-67.

33. Paterson DL, Hujer KM, Hujer AM, et al. Extended-spectrum betalactamases in Klebsiella pneumoniae blood stream isolates from seven countries: dominance and widespread prevalence of SHV and CTX-Mtype beta-lactamases. Antimicrob Agents Chemother. 2003;47(11):3554 3560

34. Smith CS, Aerts A, Saunderson P, et al. Multidrug therapy for leprosy: a game changer on the path to elimination. Lancet Infect Dis. 2017;17(9):e293-e297.

35. Remali J, Sarmin N'M, Ng CL, et al. Genomic characterization of a new endophytic "Streptomyces kebangsaanensis" identifies biosynthetic pathway gene clusters for novel phenazine antibiotic production. Peer $J$. 2017;5:e3738.

36. Philippon A, Slama P, Dény P, et al. A Structure-Based Classification of Class A $\beta$-Lactamases, a Broadly Diverse Family of Enzymes. Clin Microbiol Rev. 2016;29(1):29-57.
37. Eidam O, Romagnoli C, Dalmasso G, et al. Fragment-guided design of subnanomolar $\beta$-lactamase inhibitors active in vivo. Proc Natl Acad Sci USA. 2012;109(43):17448-17453.

38. Eidam O, Romagnoli C, Caselli E, et al. Design, synthesis, crystal structures and antimicrobial activity of sulfonamide boronic acids as $\beta$ lactamase inhibitors. J Med Chem. 2010;53(21):7852-7863.

39. Pavan FR, de Carvalho GS, da Silva AD, et al. Synthesis and AntiMycobacterium tuberculosis. Evaluation of Aza-Stilbene Derivatives. Scientific World Journal. 2011;11:1113-1119.

40. Sun D, Hurdle JG, Lee R, et al. Evaluation of flavonoid and resveratro chemical libraries reveals abyssinone II as a promising antibacterial lead. Chem Med Chem. 2012;7(9):1541-1545.

41. Snyder NJ, Tabas LB, Berry DM, et al. Structure-activity relationship of carbacephalosporins and cephalosporins: antibacterial activity and interaction with the intestinal proton-dependent dipeptide transport carrier of Caco-2 cells. Antimicrob Agents Chemother. 1997;41(8):16491657. 